\title{
Adjunctive vitamin D for the treatment of active juvenile idiopathic arthritis: An open-label, prospective, randomized controlled trial
}

\author{
TAO TANG, YU ZHANG, CHONG LUO, MINGYUE LIU, LI XU and XUEMEI TANG \\ Rheumatology and Immunology Department, Children's Hospital of Chongqing Medical University, \\ Chongqing 400014, P.R. China
}

Received January 9, 2019; Accepted September 10, 2019

DOI: $10.3892 /$ etm.2019.8133

\begin{abstract}
Vitamin D has an important immunomodulatory effect, but no trial has examined the effect of boosting serum levels of 25-hydroxyvitamin D (25OHD) in individuals with juvenile idiopathic arthritis (JIA). The aim of the present study was to assess whether vitamin D supplementation reduced disease activity and adjusted/maintained bone mass in patients with active JIA. A 24-week randomized trial was undertaken at Children's Hospital of Chongqing Medical University. Treatment-naive patients with JIA were randomly assigned (1:1) to one of two groups: Standard treatment with high dose oral cholecalciferol [2,000 IU per day; experimental group (EG)] or without supplementation [control group (CG)]. The primary outcomes were the 27-joint Juvenile Arthritis Disease Activity Score (JADAS-27 score), the Z-score for bone mineral density (BMD), and serum levels of 25OHD. A per-protocol analysis set approach was used. The Mann-Whitney U test was the main tool used for data analysis. A total of 42 participants were assigned randomly to the EG $(n=20)$ or the $C G(n=22)$; of these, $36(n=18$ and $n=18$, respectively) were included in per-protocol analysis. After 24 weeks, the mean level of 25OHD in the EG was higher than that in the $\mathrm{CG}(\mathrm{P}<0.05)$. At the end of the intervention, there were no clear differences between the two groups in terms of BMD or JADAS-27 score (both $\mathrm{P}>0.05$ ). Cholecalciferol supplementation (2000 IU/day) for 24 weeks raised serum levels of $25 \mathrm{OHD}$ in JIA patients but did not reduce disease activity or improve BMD (registration no. ChiCTR-INR-16009235; Date of Registration: 2016-10-12).
\end{abstract}

Correspondence to: Dr Xuemei Tang, Rheumatology and Immunology Department, Children's Hospital of Chongqing Medical University, 136 Second Zhongshan Road, Yuzhong, Chongqing 400014, P.R. China

E-mail: tangxuemei2008@163.com

Key words: disease activity, bone mineral density, juvenile idiopathic arthritis, vitamin D, 25-hydroxyvitamin D

\section{Introduction}

Juvenile idiopathic arthritis (JIA) is a heterogeneous group of diseases characterized by arthritis of unknown origin and disease onset before the age of 16 years; thus JIA causes childhood disability $(1,2)$. As a result of chronic inflammation, patients with JIA suffer from joint pain, swelling, restricted range of motion and joint deformity (1). Severe extra-articular abnormalities may also accompany this disease, including uveitis, which occurs more frequently in antinuclear antibody (ANA)-positive patients and can lead to blindness, and macrophage activation syndrome (MAS), a complication of sJIA that can lead to multi-organ insufficiency and even mortality $(3,4)$

Previous studies indicated that vitamin D played an important role in maintaining both the skeletal and immune systems $(5,6)$. Vitamin D regulates the innate and adaptive immune systems by activating the vitamin $\mathrm{D}$ receptor, which is distributed widely on immune cells $(7,8)$. Consequently, vitamin $\mathrm{D}$ deficiency is associated with autoimmune diseases such as diabetes, rheumatoid arthritis, systemic lupus erythematosus, and JIA (9-20). A previous study reported suboptimal vitamin D levels in JIA patients (13). Moreover, studies suggest that 25-hydroxyvitamin D (25OHD) is negatively associated with JIA disease activity $(14,15)$. Studies in mouse models of rheumatoid arthritis confirmed that the vitamin $\mathrm{D}$ receptor plays an important role in limiting the inflammatory phenotype (21), although other studies refute this (16).

Previous work showed that patients with JIA, especially those with higher disease activity, have low bone mass (18). However, although several studies have examined the role of vitamin D in JIA (18-22), the immunomodulatory effects of supplementation with vitamin D have not been previously investigated. The aim of the present study was to examine the effect of cholecalciferol supplementation on serum vitamin D levels, disease activity scores, and bone mineral density in patients with JIA.

\section{Patients and methods}

The present study was a randomized, comparative, monocentric trial (Chinese Clinical Trial Registry no. ChiCTR-INR-16009235) conducted at the Children's 
Hospital of Chongqing Medical University (Chongqing, China). From October 20, 2016 to February 15, 2018, 42 treatment-naive JIA patients diagnosed at the Rheumatology and Immunology Inpatient Department and followed at the Outpatient Clinic, Children's Hospital of Chongqing Medical University, were enrolled. All participants (6.9 \pm 3.1 years), or their guardians, provided written informed consent. The study was approved by the Institutional Review Board at Children's Hospital of Chongqing Medical University. Patients were selected using a table of random numbers. All patients met the 2001 International League of Associations for Rheumatology classification criteria (23). Participants were randomized into two parallel groups using a random number table: i) Vitamin D (Xiamen Lipin Pharmaceutical Co., Ltd.) supplementation [2000 IU per day; experimental group (EG)]; and ii) no treatment [control group (CG)]. Both groups received standard therapy [glucocorticoids $(0.5-1 \mathrm{mg} / \mathrm{kg} / \mathrm{d})$, non-steroidal anti-inflammatory drugs (30-40 mg/kg/d), methotrexate $(10-15 \mathrm{mg} / \mathrm{m} 2 / \mathrm{w})$, or sulfasalazine (30-50 mg/kd/d)]. None of the patients had taken vitamin D for at least 3 months prior to entry into the study. Exclusion criteria were as follows: A history of kidney stones, hypercalciuria, intestinal malabsorption, primary cardiovascular disease, lung disease, blood disease, liver disease, a history of using drugs that inhibit bone resorption, a history of allergy to vitamin D, and refusal to participate in the study. Patients treated with methylprednisolone, biological agents, or cyclophosphamide were also excluded. Demographic data, disease duration, findings upon physical examination, erythrocyte sedimentation rates, parathyroid hormone levels, disease activity, bone mineral density (BMD), and serum 25OHD levels were evaluated. The 27-joint juvenile arthritis disease activity score (JADAS-27) (24) was used as a measure of disease activity. Venous blood was collected $(5 \mathrm{ml})$ at room temperature and centrifuged for $10 \mathrm{~min}$ at $1409 \mathrm{x} \mathrm{g}$, and the serum was separated from the hemocytes. A blood sample of $150 \mu \mathrm{l}$ was taken. Deproteinization was performed with saturated zinc sulfate solution (CAS: 7733-02-0; Shanghai Jingke Scientific Instrument Co., Ltd.), acetonitrile (CAS: 75-05-8; Tokyo Chemical Industry UK Ltd.) and dehydrated alcohol. 25OHD extraction from the serum was conducted with hexane, and the hexane was evaporated using nitrogen gas, then it was dissolved by methanol solution and detected using high-performance liquid chromatography apparatus (APS80-16D; AUPOS), with a flow rate of $0.5 \mathrm{ml} / \mathrm{min}$. The Z-score, calculated from dual-energy X-ray absorptiometry, was used as a measure of bone mineral density (BMD; Delphi-A system; Hologic, Inc.). The normal vitamin D range is 75 to $250 \mathrm{nmol} / \mathrm{l}$. Vitamin D insufficiency was defined as serum levels between $<75 \mathrm{nmol} / \mathrm{l}$ and $50 \mathrm{nmol} / \mathrm{l}$. Levels lower than $50 \mathrm{nmol} / 1$ were classified as vitamin D deficiency (25). The cumulative doses of glucocorticoids were also compared in 6 months of each group to identify differences. The primary outcomes were evaluated at weeks 0,12 , and 24 . Safety and tolerability were also assessed at every visit.

Statistical analysis. Student's t-test was used to analyze parametric data and the Mann-Whitney U test was used for non-parametric data. The one-sample Kolmogorov-Smirnov test was used to check data distribution. Differences within each group were compared using one-way analysis of variance
Table I. One-sample Kolmogorov-Smirnov test parameters at baseline, week 12, and week 24 .

\begin{tabular}{lrrr} 
A, Baseline & & & \\
\hline Parameter & Mean & SD & P-value \\
\hline Experimental group $(\mathrm{n}=18)$ & & & \\
25OHD, nmol/1 & 33.29 & 12.87 & 0.200 \\
JADAS-27 & 16.19 & 9.57 & 0.048 \\
Z-score & -1.10 & 1.48 & 0.200 \\
Control group (n=18) & & & \\
25OHD, nmol/1 & 51.60 & 34.77 & 0.041 \\
JADAS-27 & 14.31 & 6.22 & 0.200 \\
Z-score & -1.15 & 0.96 & 0.200 \\
\hline
\end{tabular}

B, Week 12

\begin{tabular}{lrrr}
\hline Parameter & Mean & SD & P-value \\
\hline Experimental group (n=18) & & & \\
25OHD, nmol/1 & 65.19 & 15.52 & 0.200 \\
JADAS-27 & 4.56 & 5.89 & 0.022 \\
Z-score & -0.68 & 1.47 & 0.200 \\
Control group (n=18) & & & \\
25OHD, nmol/1 & 43.46 & 15.60 & 0.200 \\
JADAS-27 & 5.51 & 7.51 & 0.012 \\
Z-score & -0.95 & 0.77 & 0.200 \\
\hline
\end{tabular}

C, Week 24

\begin{tabular}{lrrr}
\hline Parameter & Mean & \multicolumn{1}{c}{ SD } & P-value \\
\hline Experimental group $(\mathrm{n}=18)$ & & & \\
25OHD, nmol/1 & 69.25 & 15.52 & 0.200 \\
JADAS-27 & 0.94 & 2.31 & 0.000 \\
Z-score & -0.61 & 1.29 & 0.200 \\
Control Group (n=18) & & & \\
25OHD, nmol/1 & 38.83 & 13.12 & 0.040 \\
JADAS-27 & 1.06 & 2.82 & 0.000 \\
Z-score & -0.97 & 0.84 & 0.200 \\
\hline
\end{tabular}

Null-hypothesis = normal distribution. BMD, bone mineral density; JADAS-27, 27-joint Juvenile Arthritis Disease Activity Score; 25OHD, 25-hydroxyvitamin D.

(parametric data) or related-samples Friedman's two-way analysis of variance by rank (non-parametric data). Multiple comparison between the groups was performed using the Friedman two-way analysis of variance (ANOVA) by Ranks Test. Statistical analysis was performed using SPSS version 23.0 for Mac OS (IBM Corp.). $\mathrm{P}<0.05$ was considered to indicate a statistically significant difference. Continuous variables with a normal distribution are reported as the mean \pm standard deviation (SD) and non-normally distributed variables are reported as the median (interquartile range). 
Table II. Baseline parameters of JIA patients according to treatment group.

\begin{tabular}{|c|c|c|c|}
\hline Parameter & Experimental group $n=18$ & Control group $n=18$ & P-value \\
\hline Age, years & $7.6(4.3)$ & $6.3(2.9)$ & 0.31 \\
\hline Season & & & 0.66 \\
\hline Summer $(\%)$ & 22 & 11 & \\
\hline Other $(\%)$ & 78 & 89 & \\
\hline Type & & & 0.71 \\
\hline SJIA $(\%)$ & 27 & 33 & \\
\hline Non-SJIA (\%) & 73 & 67 & \\
\hline Sex & & & 0.49 \\
\hline Male $(\%)$ & 44 & 28 & \\
\hline Female $(\%)$ & 56 & 62 & \\
\hline 25OHD, nmol/1 & $33.29(12.87)$ & $51.60(34.77)$ & 0.15 \\
\hline JADAS-27 & $16.19(9.57)$ & $14.31(6.22)$ & 0.84 \\
\hline Z-score & $-1.10(1.48)$ & $-1.15(0.96)$ & 0.94 \\
\hline
\end{tabular}

Data are expressed as the mean $( \pm \mathrm{SD})$ or \%. BMD, bone mineral density; JADAS-27, 27-joint Juvenile Arthritis Disease Activity Score; 25OHD, 25-hydroxyvitamin D.

\section{Results}

Forty-four subjects met the inclusion criteria. Two of these refused assignment to the EG. The remaining patients were assigned randomly to the $\mathrm{CG}(\mathrm{n}=22)$ or the $\mathrm{EG}(\mathrm{n}=20)$. A total of six patients withdrew for personal reasons or were lost to follow-up. Finally, 36 subjects completed the trial and were included in the analysis ( $\mathrm{n}=18$ per group).

Data were collected at baseline and at week 12 and 24. All outcome measures, except the Z-score for BMD, showed a non-normal distribution (Table I); therefore, the Mann-Whitney U test was the main tool for data analysis.

25OHD. The baseline data for both groups are presented in Table II. The anthropometric, clinical disease parameters were similar for both groups (all $\mathrm{P}>0.05$ ). The mean serum level of 25OHD in the EG at baseline was $33.29 \mathrm{nmol} / 1 \mathrm{vs}$. $51.60 \mathrm{nmol} / 1$ in the $\mathrm{CG}(\mathrm{P}=0.15)$. Overall, the prevalence of vitamin $\mathrm{D}$ insufficiency/deficiency in all JIA patients at baseline was $89.9 \%$.

After 12 and 24 weeks of supplementation, serum 25OHD levels in EG patients were higher than those in CG patients (week 12: 65.19 vs. 43.46, respectively; $\mathrm{P}<0.05$; week 24: 69.25 vs. 38.83 , respectively; $\mathrm{P}<0.05$ ). After 24 weeks, $39.9 \%$ of patients in the EG had serum 25OHD levels $>75 \mathrm{nmol} / \mathrm{l}$; by contrast, $0 \%$ of patients in the $\mathrm{CG}(\mathrm{P}<0.05)$ reached these levels. Moreover, only $18.75 \%$ of patients in the $\mathrm{CG}$ reached 25OHD levels $>50 \mathrm{nmol} / 1$, compared with $88.89 \%$ in the EG after 24 weeks (Fig. 1).

JADAS-27 score. The JADAS-27 score for 18 patients who received vitamin $\mathrm{D}$ supplementation was no better than that in the CG at week 12 or at the end of the study [week 12: 4.56 vs. 4.56, respectively, $\mathrm{P}>0.05$; week 24: 0.94 vs. 1.06, respectively; $\mathrm{P}>0.05]$. They all exhibited good outcomes in terms of clinical characteristics as $38.89 \%$ of the patients in the EG recorded a

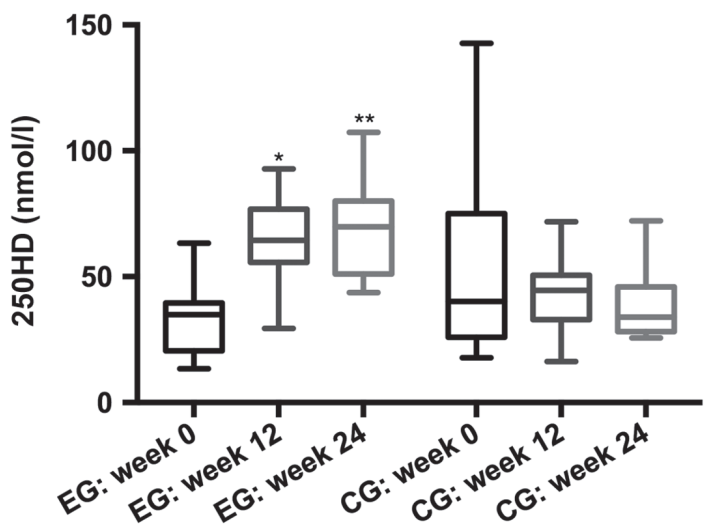

Figure 1. Serum 25OHD levels in the EG and CG. After 12 and 24 weeks of supplementation, serum 25OHD levels in EG patients were higher than those in $\mathrm{CG}$ patients $(\mathrm{P}<0.05)$. 25OHD, 25-hydroxyvitamin $\mathrm{D}$; CG, control group; EG, experimental group; ${ }^{*} \mathrm{P}<0.05$; EG vs. CG after 12 weeks; ${ }^{* *} \mathrm{P}<0.05$; EG vs. CG after 24 weeks.

JADAS-17 score of 0 at week 12 , whereas $83.33 \%$ achieved this score at week 24; and percentages in the CG were 37.50 and $83.33 \%$, respectively (Fig. 2).

$B M D$. The one-sample Kolmogorov-Smirnov test revealed that BMD data showed a normal distribution. The one-way analysis of variance and related-samples Friedman's two-way ANOVA by rank tests showed no significant differences in the $\mathrm{Z}$-score for BMD in either group at week $24(\mathrm{P}>0.05)$. There were no significant differences between the EG and CG at 12 or 24 weeks (-0.68 vs. -0.95 , respectively; $\mathrm{P}>0.05$, at week 12 ; -0.61 vs. -0.97 , respectively; $P>0.05$, at week 24 ; Fig. 3 ).

In the present study, five patients with systemic JIA ( $\mathrm{nEG}=3, \mathrm{nCG}=4)$ were treated with glucocorticoids (Table III). The cumulative doses of glucocorticoids in 6 months showed no significant differences between the two groups (2249.17 vs. 2117.50 , respectively; $\mathrm{P}>0.05$ ) 


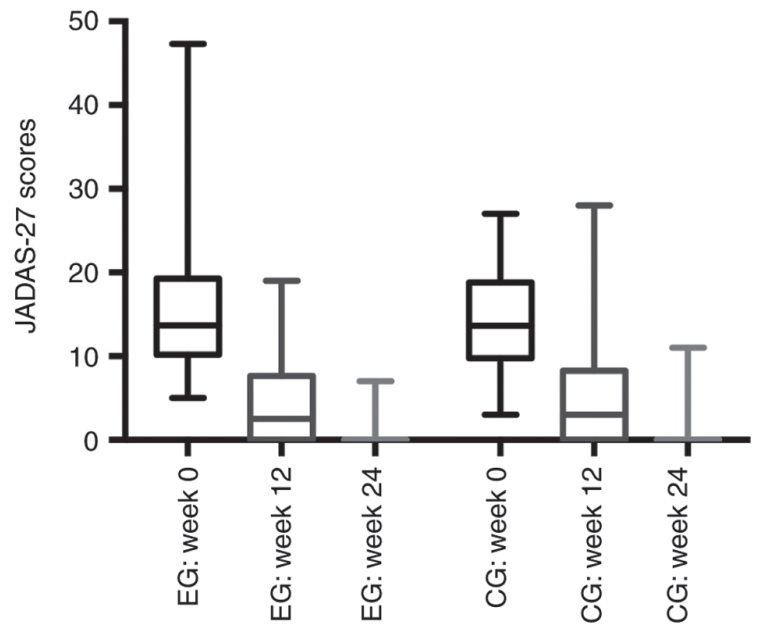

Figure 2. JADAS-27 scores for the EG and CG. There was no significant difference in the JADAS-27 scores for the two groups after 12 or 24 weeks of supplementation with 25OHD. 250HD, 25-hydroxyvitamin D; CG, control group; EG, experimental group; JADAS-27, 27-joint Juvenile Arthritis Disease Activity Score.

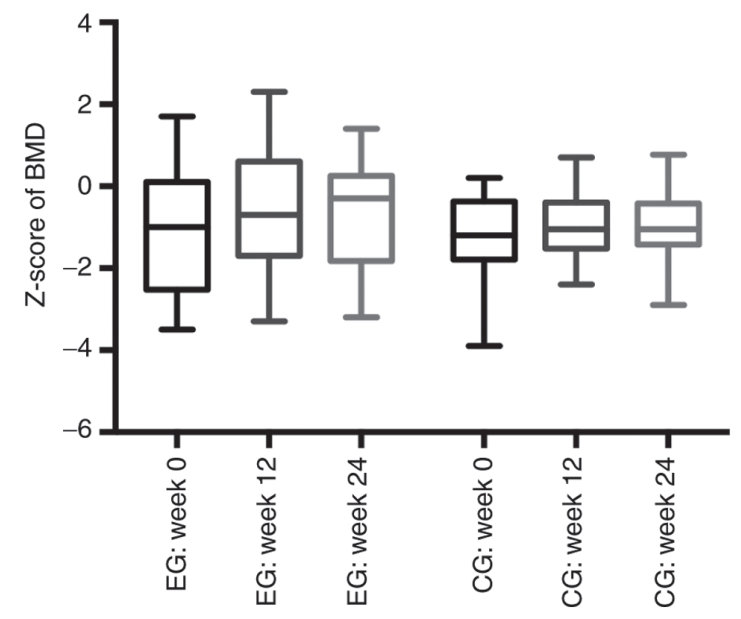

Figure 3. Z-scores for bone mineral density for the EG and CG. There was no significant difference in the Z-scores for either group after 12 and 24 weeks of supplementation with 25OHD. 25OHD, 25-hydroxyvitamin D; CG, control group; EG, experimental group.

Above all the patients, only 4 patients' serum 25OHD concentrations of $<80 \mathrm{nmol} / \mathrm{l}$; half of these $(\mathrm{n}=2)$ showed a significant benefit in terms of BMD $(\mathrm{P}<0.05)$.

Supplementation was well tolerated, with no safety issues for either group. No serious adverse events were recorded, and no patients had kidney calculi, liver damage, hypercalcemia, or gastrointestinal symptoms.

\section{Discussion}

Taken together, the findings presented above suggest that cholecalciferol supplementation (2,000 IU per day) for 24 weeks led to a significant increase in serum $250 H D$ levels in JIA patients but did not reduce disease activity or improve BMD.

The number of participants was small. Only 60 patients with JIA are diagnosed at the Children's Hospital of Chongqing Medical University each year, and the number of
Table III. Glucocorticoid therapy of JIA patients according to treatment group.

\begin{tabular}{lll}
\hline Number & Group & $\begin{array}{c}\text { Cumulative dose mg, } \\
(6 \text { months })\end{array}$ \\
\hline 1 & EG & 1617.5 \\
2 & EG & 2850 \\
3 & EG & 2280 \\
4 & CG & 3132.5 \\
5 & CG & 2585 \\
6 & CG & 1215 \\
7 & CG & 1537.5 \\
\hline
\end{tabular}

EG: Experimental Group, CG: Control Group, Cumulative dose: Cumulative dose of glucocorticoids in 6 months.

treatment-naive patients is even lower than this. A single-center study may also suffer from selection bias. Despite these limitations, to the best of our knowledge, the present work is the first to examine the outcomes of cholecalciferol supplementation (2,000 IU per day) in Southwestern China. The results of the present study revealed that 2,000 IU per day of vitamin D supplementation was safe. Future work examining the effects of high dose supplementation will be needed to clarify the relationship between JIA and vitamin D.

A previous study showed that the immunomodulatory effects of cholecalciferol were only observed in individuals for whom the 25OHD increased to more than $100 \mathrm{nmol} / 1$ and any beneficial effect disappeared when serum levels dropped below $100 \mathrm{nmol} / \mathrm{l}$ (26). Other reports revealed that the anti-inflammatory benefits of vitamin $\mathrm{D}$ were due to increased expression of toll-like receptor 2 (TLR2) by peripheral blood mononuclear cells and reduced TLR2-mediated production of tumor necrosis factor- $\alpha$, interleukin- 6 , and interferon- $\gamma$. These cytokines promoted local inflammation, leading to tissue damage and expression of auto-antigens, which then triggered autoreactive immune responses in JIA $(1,27,28)$.

In China, the daily amount of vitamin $\mathrm{D}$ recommended by the Chinese Nutrition Society is $400 \mathrm{IU} / \mathrm{d}(10 \mu \mathrm{g} / \mathrm{d})(29)$; the tolerable upper intake level is $1800 \mathrm{IU} / \mathrm{d}(45 \mu \mathrm{g} / \mathrm{d})$. In the present study a safe oral dose was defined as 2,000 IU/d (30). Therefore, after week 24 of supplementation, serum 25OHD reached $100 \mathrm{nmol} / \mathrm{l}$ only in one patient. This result may be due to insufficient vitamin D supplementation. This is supported by a study of systemic lupus erythematosus patients who received 50,000 IU/week (19).

The lack of change in BMD noted between the groups may be because serum 25OHD levels were below the functional threshold. According to some reports, serum 25OHD concentrations of more than $80 \mathrm{nmol} / \mathrm{l}$ facilitate calcium absorption in the intestine $(31,32)$. In the present study, only four patients reached this level; half of these $(n=2)$ showed a significant benefit in terms of BMD $(\mathrm{P}<0.05)$.

Disease activity in each group decreased significantly after 12 weeks, but differences of JADAS-27 between and within the two groups were not clear. The plasma half-life of $25 \mathrm{OHD}$ is 3 weeks (33). Plasma levels of 25OHD stabilize after three to 
four half-lives (about 12 weeks); however, it was not possible to reduce the follow-up period in the present study to detect potential differences before this time. A tool that can measure disease activity more accurately may be needed for future studies.

It is important not to ignore the fact that Glucocorticoids/ methylprednisolone used in systemic JIA may affect the outcome of the present study. Because JIA is a heterogeneous group of diseases, usually only glucocorticoids/methylprednisolone are used for the treatment of systemic JIA, which resembles more an auto-inflammatory disease than an autoimmune disease (34). Treatment may have a strong effect on BMD and JIA disease activity indexes. However, in the present study, five patients with systemic JIA $(\mathrm{nEG}=3, \mathrm{nCG}=4)$ were treated with glucocorticoids still showed no statistically significant difference (2249.17 vs. 2117.50, respectively; $\mathrm{P}>0.05$ )

High dose, randomized, double-blinded, controlled studies with a greater number of subjects are required to fully examine the benefits of vitamin D and it's in vivo role in JIA patients. It would be interesting to examine serum levels of 25OHD and disease activity changes in patients with JIA during and after pregnancy (35).

\section{Acknowledgements}

The authors would like to thank Dr Zhang Zhiyong, Dr An Yunfei and Dr Ding Yuan for aiding in the selection of participants (all, Department of Rheumatology and Immunology, Children's Hospital of Chongqing Medical University).

\section{Funding}

The project was supported by Children's Hospital of Chongqing Medical University and Chongqing City Health and Family Planning Committee (grant no. 2016MSXM033).

\section{Availability of data and materials}

The datasets used and/or analyzed during the current study are available from the corresponding author on reasonable request.

\section{Authors' contributions}

TX, TT, ZY and LC designed and supervised the study. TT, LM, XL and TX performed the experiments, with help from all the other authors. TT, ZY and LC analyzed the data, while TT and TX wrote the manuscript. All authors read and approved the final form of manuscript.

\section{Ethics approval and consent to participate}

The experimental protocol was established, according to the ethical guidelines of the Helsinki Declaration and was approved by the Institutional Review Board of Children's Hospital of Chongqing Medical University. Written informed consent was obtained from individual or guardian participants.

\section{Patient consent for publication}

In the present clinical trial, the patient's guardian signed a written informed consent to publish any relevant data.

\section{Competing interests}

The authors declare that they have no competing interests.

\section{References}

1. Prakken B, Albani S and Martini A: Juvenile idiopathic arthritis. Lancet 377: 2138-2149, 2011.

2. Martini A and Lovell DJ: Juvenile idiopathic arthritis: State of the art and future perspectives. Ann Rheum Dis 69: 1260-1263, 2010.

3. Bracaglia C, Prencipe G and Benedetti FD: Macrophage Activation Syndrome: Different mechanisms leading to a one clinical syndrome. Pediatr Rheumatol Online J 15: 5, 2017.

4. Tappeiner C, Klotsche J, Sengler C, et al: Risk factors and biomarkers for the occurrence of uveitis in JIA: Data from the Inception Cohort of Newly diagnosed patients with Juvenile Idiopathic Arthritis (ICON-JIA) study. Arthritis Rheumatol, 2018. [J]. https://doi.org/10.1002/art.40544.

5. DeLuca HF: Overview of general physiologic features and functions of vitamin D. Am J Clin Nutr 80 (Suppl): 1689S-1696S, 2004.

6. White JH: Vitamin D metabolism and signaling in the immune system. Rev Endocr Metab Disord 13: 21-29, 2012.

7. Kamen DL and Tangpricha V: Vitamin D and molecular actions on the immune system: Modulation of innate and autoimmunity. J Mol Med (Berl) 88: 441-450, 2010.

8. White JH: Vitamin D metabolism and signaling in the immune system. Rev Endocr Metab Disord 13: 21-29, 2012.

9. Maddaloni E, Cavallari I, Napoli N and Conte C: Vitamin D and diabetes mellitus. Front Horm Res 50: 161-176, 2018.

10. Jeffery LE, Raza K and Hewison M: Vitamin D in rheumatoid arthritis-towards clinical application. Rheumatol 12: 201-210, 2016.

11. Iruretagoyena M, Hirigoyen D, Naves R and Burgos PI: Immune response modulation by vitamin D: Role in systemic lupus erythematosus. Front Immunol 6: 513, 2015.

12. Lima GL, Paupitz J, Aikawa NE, Takayama L, Bonfa E and Pereira RM: Vitamin D supplementation in adolescents and young adults with juvenile systemic lupus erythematosus for improvement in disease activity and fatigue scores: A randomized, double-blind, placebo-controlled trial. Arthritis Care Res (Hoboken) 68: 91-98, 2016.

13. Finch SL, Rosenberg AM and Vatanparast H: Vitamin D and juvenile idiopathic arthritis. Pediatr Rheumatol Online J 16: 34, 2018.

14. Bouaddi I, Rostom S, El Badri D, Hassani A, Chkirate B, Abouqal R, Amine B and Hajjaj-Hassouni N: Vitamin D concentrations and disease activity in Moroccan children with juvenile idiopathic arthritis. BMC Musculoskelet Disord 15: 115, 2014.

15. Çomak E, Doğan ÇS, Uslugökçeoğlu A, et al: Association between vitamin $\mathrm{D}$ deficiency and disease activity in juvenile idiopathic arthritis. Turk J Pediatr 71 (Suppl 3): 702-702, 2013.

16. Pelajo CF, Lopez-Benitez JM, Kent DM, Price LL, Miller LC and Dawson-Hughes B: 25-hydroxyvitamin D levels and juvenile idiopathic arthritis: Is there an association with disease activity? Rheumatol Int 32: 3923-3929, 2012.

17. Tang T, Tang X, Xu L, Huang Y, Zeng J and Li Q: Evaluation of bone mass in children and young adults with juvenile idiopathic arthritis. Clin Exp Rheumatol 33: 758-764, 2015.

18. Lima GL, Paupitz J, Aikawa NE, et al: A randomized double-blind placebo-controlled trial of vitamin D supplementation in adolescents and young adults with Juvenile-onset SLE: Improvement in disease activity and fatigue scores. Arthritis Care Res (Hoboken) 68: 91, 2015.

19. Ellis JA, Scurrah KJ, Li YR, Ponsonby AL, Chavez RA, Pezic A, Dwyer T, Akikusa JD, Allen RC, Becker ML, et al: Epistasis amongst PTPN2 and genes of the vitamin D pathway contributes to risk of juvenile idiopathic arthritis. J Steroid Biochem Mol Biol 145: 113-120, 2015.

20. Çomak E, Doğan CS, Gökçeoglu AU, et al: Evaluation of vitamin D levels in children with juvenile idiopathic arthritis. Çocuk Sagligi Hastalik Derg 55: 191-196, 2012.

21. Zwerina K, Baum W, Axmann R, Heiland GR, Distler JH, Smolen J, Hayer S, Zwerina J and Schett G: Vitamin D receptor regulates TNF-mediated arthritis. Ann Rheum Dis 70: 1122-1129, 2011.

22. Stagi S, Bertini F, Cavalli L, Matucci-Cerinic M, Brandi ML and Falcini F: Determinants of vitamin D levels in children, adolescents, and young adults with juvenile idiopathic arthritis. J Rheumatol 41: 1884-1892, 2014. 
23. Petty RE, Southwood TR, Manners P, Baum J, Glass DN, Goldenberg J, He X, Maldonado-Cocco J, Orozco-Alcala J, Prieur AM, et al; International League of Associations for Rheumatology: International League of Associations for Rheumatology classification of juvenile idiopathic arthritis: Second revision, Edmonton, 2001. J Rheumatol 31: 390-392, 2004.

24. Consolaro A, Ruperto N, Bazso A, Pistorio A, Magni-Manzoni S, Filocamo G, Malattia C, Viola S, Martini A and Ravelli A; Paediatric Rheumatology International Trials Organisation: Development and validation of a composite disease activity score for juvenile idiopathic arthritis. Arthritis Rheum 61: 658-666, 2009.

25. Holick MF, Binkley NC, Bischoff-Ferrari HA, Gordon CM, Hanley DA, Heaney RP, Murad MH and Weaver CM; Endocrine Society: Evaluation, treatment, and prevention of vitamin D deficiency: An Endocrine Society clinical practice guideline. J Clin Endocrinol Metab 96: 1911-1930, 2011.

26. Ojaimi S, Skinner NA, Strauss BJ, Sundararajan V, Woolley I and Visvanathan K: Vitamin D deficiency impacts on expression of toll-like receptor- 2 and cytokine profile: A pilot study. J Transl Med 11: 176, 2013

27. Jarvis JN, Jiang K, Frank MB, Knowlton N, Aggarwal A, Wallace CA, McKee R, Chaser B, Tung C, Smith LB, et al: Gene expression profiling in neutrophils from children with polyarticular juvenile idiopathic arthritis. Arthritis Rheum 60: 1488-1495, 2009.
28. Gregorio A, Gambini C, Gerloni V, Parafioriti A, Sormani MP, Gregorio S, De Marco G, Rossi F, Martini A, et al: Lymphoid neogenesis in juvenile idiopathic arthritis correlates with ANA positivity and plasma cells infiltration. Rheumatology (Oxford) 46:308-313, 2007.

29. Chinese Nutrition Society. Dietary nutrient intake for Chinese residents (M). (in Chinese) China light industry press, 2010.

30. John N: Hathcock. 3rd edition. Vitamin and Mineral Safety, pp33-36, 2014.

31. Heaney RP, Dowell MS, Hale CA and Bendich A: Calcium absorption varies within the reference range for serum 25-hydroxyvitamin D. J Am Coll Nutr 22: 142-146, 2003.

32. Abrams SA, Hicks PD and Hawthorne KM: Higher serum 25-hydroxyvitamin D levels in school-age children are inconsistently associated with increased calcium absorption. J Clin Endocrinol Metab 94: 2421-2427, 2009.

33. Zerwekh JE: Blood biomarkers of vitamin D status. Am J Clin Nutr 87: 1087S-1091S, 2008. [J].

34. Mellins ED, Macaubas C and Grom AA: Pathogenesis of systemic juvenile idiopathic arthritis: Some answers, more questions. Nat Rev Rheumatol 7: 416-426, 2011. [J].

35. Ursin K, Lydersen S, Skomsvoll JF and Wallenius M: Disease Activity of Juvenile Idiopathic Arthritis during and after Pregnancy: A Prospective Multicenter Study. J Rheumatol 45: 257-265, 2018. 